\title{
LA MIGRACIÓN DE RUMANIA: NUEVOS Y ANTIGUOS ESCENARIOS PARA LA MOVILIDAD ${ }^{1}$ THE MIGRATION OF ROMANIA: OLD AND NEW SCENARIOS FOR MOBILITY
}

\author{
Emiliana Baldoni \\ Istat (Istituto Nazionale di Statistica) \\ emiliana.baldoni@fastwebnet.it
}

Recibido: $7 / 10 / 2010$

Aceptado: 15/12/2010

\begin{abstract}
Resumen
Este trabajo analiza el fenómeno de la movilidad desde Rumanía (interna, internacional y transfronteriza), recorriendo las fases históricas y las principales características antes y después de que este país se integrara en la Unión Europea. El "sistema de inmigración de Rumanía" tiene sus raices en la movilidad interna del período Socialista, y actualmente se caracteriza por el predominio de los movimientos circulares que, como en el caso italiano, coexisten sin embargo con una marcada tendencia a la estabilización.

Palabras clave: sistema de migración de Rumanía, migración circular, movilidad interna, transnacionalismo, movilidad intra-europea
\end{abstract}

\begin{abstract}
This paper analyzes the phenomenon of mobility from Romania (internal, international and cross-border) tracing the historical phases and the main characteristics before and after this country entered the European Union. The "Romanian immigration system" has taken root in the internal mobility of the Socialist period and now is characterized by the predominance of cir-
\end{abstract}

\footnotetext{
${ }^{1}$ Presentado en el Seminario Internacional Migraciones Circulares, el retorno como estrategia de desarrollo en los países de Origen, celebrado en Alicante, del 6 al 8 de octubre de 2010, organizado por el Instituto Interuniversitario de Desarrollo Social y Paz, con la colaboración del Ministerio de Ciencia e Innovación (Ref. CSO2009-07331-E) y de la Conselleria d'Educació de la Generalitat Valenciana (Ref. AORG/2010/124)
} 
cular movements that, as in the Italian case, however, coexist with strong trends to stabilization.

Keywords: Romanian migration system; circular migration; internal mobility; transnationalism; intra-EU mobility

\section{LA ENTRADA DE RUMANÍA EN LA UNIÓN EUROPEA: ¿QUÉ MOVILIDAD?}

El proceso de ampliación hacia el Este, primero con la anexión de Chipre, Estonia, Letonia, Lituania, Malta, Polonia, República Checa, Eslovaquia, Eslovenia y Hungría en 2004, y a continuación, de Rumanía y Bulgaria en 2007, dio lugar a un traslado de los límites de la Unión Europea y a la configuración de un nuevo sistema geo-político (aún no definitivo). Las implicaciones de este proceso sobre la movilidad internacional son muy importantes.

Para los países que más han sufrido las restricciones de los regímenes comunistas, la extensión de la libre circulación, derecho previsto en el artículo 39 del Tratado CE y regido por la Directiva 2004/38/CE, representa el fin del aislamiento y la oportunidad de acceder al mundo occidental, prohibido y mitificado por más de una generación. Al mismo tiempo, la metamorfosis de las fronteras obliga a los países de Europa Central y Oriental a convertirse en una nueva "puerta de entrada" en la Unión Europea: una cortina de múltiples formas de vigilancia, nacida un poco más al Este del Telón de Acero para filtrar a las poblaciones excluidas de la libre movilidad (Gambino, Sacchetto, 2007: 18).

En la víspera de la entrada de Rumania y Bulgaria, se extendieron temores irracionales de "invasiones de masas" (sabiamente impulsados por algunas fuerzas políticas), creando alarma y ansiedad en la opinión pública (D’Angelo, 2008). Así, mientras que la UE hacía hincapié en que la movilidad interna era demasiado baja, los "antiguos" Estados miembros (con excepción de Suecia y Finlandia) optaban por la adopción de medidas transitorias que preveían restricciones en el acceso al mercado de trabajo de los rumanos y búlgaros hasta un período máximo de siete años ${ }^{2}$. En particular, las disposiciones transitorias establecían que en los dos primeros años después de la adhesión, el acceso a los mercados de trabajo de los estados miembros de la UE-25 dependía de las legislaciones nacionales, así como de los acuerdos bilaterales con Bulgaria y Rumania (en términos prácticos, se necesitaba un permiso de trabajo) ${ }^{3}$.

\footnotetext{
${ }^{2}$ Las medidas transitorias se aplican a todos los ciudadanos búlgaros y rumanos que desean un contrato de trabajo o buscar empleo en un país de la UE; no se refieren, sin embargo, a los que desean residir en la UE para fines de estudios o para establecer una actividad por cuenta propia.

${ }^{3}$ Las disposiciones transitorias establecen que cada Estado miembro podrá seguir aplicando medidas nacionales por un periodo máximo de tres años más allá de los dos pri-
} 
Italia, con la circular del Ministerio del Interior n. 7881 de diciembre 2009 ("Ciudadanos de la República de Bulgaria y Rumanía - Normas para el acceso al mercado de trabajo") ha decidido mantener sin cambios, también durante el año 2010, las disposiciones transitorias ya adoptadas en los años 2007, 2008 y 2009. Quedan, por tanto, ciertas restricciones de acceso al mercado de trabajo. Para la contratación de trabajadores procedentes de Rumanía y Bulgaria, de hecho, se necesita la autorización del "Portal Único para la Inmigración" del Ministerio, con la excepción de ciertos sectores como agricultura, turismo, hoteles, trabajo doméstico y asistencia personal, sector de la construcción, ingeniería industrial, ingeniería de alimentos, sector de gestión y trabajo estacional, para los cuales está previsto un régimen de libre acceso ${ }^{4}$. En estos sectores, donde se registra una fuerte demanda, los contratos de trabajo podrán ser adjudicados directamente, sin pedir autorización, en igualdad de condiciones con el resto de los ciudadanos.

Por otro lado, como subrayan Gambino y Bolsa (2007: 21-22), la entrada de los países de Europa central y oriental ha dado lugar a una renovación en la división internacional del trabajo, que se caracteriza por un intercambio continuo y desigual entre los empleadores y los trabajadores locales y extranjeros. En Rumania, por ejemplo, en la última década, los flujos de inversión extranjera han crecido de manera exponencial, favorecidos por la disponibilidad de mano de obra cualificada a bajo costo, en un marco económico general desregulado y por las perspectivas de revalorización del capital asociada a la expansión al Este de la zona Euro. A cambio, los trabajadores rumanos en Italia permanecen principalmente en empleos poco cualificados y remunerados, y no son infrecuentes en algunos sectores casos de explotación laboral.

De hecho, a pesar de las disposiciones transitorias, en los últimos años el número de rumanos en Italia (y en España) se ha más que duplicado. ¿Se trata, como algunos afirman, de flujos destinados a agotarse en el corto plazo? ¿Los factores push están perdiendo su relevancia en el contexto de la crisis

meros después de la adhesión de Rumanía y Bulgaria. La libre circulación de trabajadores debe producirse después de cinco años, es decir desde el 1 de enero de 2012. Sin embargo, los países miembros pueden solicitar la autorización de la Comisión para seguir aplicando medidas transitorias durante dos años más, pero sólo en caso de perturbación grave del mercado de trabajo nacional. A partir de 2014 -siete años después de la adhesión de los dos países- habrá plena libertad de circulación de los trabajadores en toda la UE.

${ }^{4}$ La concesión de la autorización no está aún sujeta a limitaciones particulares o a la verificación de requisitos específicos, ni siquiera están previstas sanciones en caso de falta de demanda. El único inconveniente es que, sin esta autorización, a los nuevos ciudadanos de la UE empleados en sectores no liberalizados se les puede negar la solicitud de registro en los municipios de residencia (Nanni, 2008). 
económica mundial? Con la eliminación definitiva de las restricciones a la movilidad, ¿los movimientos circulares prevalecerán sobre la tendencia hacia la estabilización?

Aunque en Rumania los movimientos migratorios hayan desempeñado un papel fundamental en la transformación social y económica del país, sólo en los últimos tiempos se han convertido en objeto de interés y de reflexión para las ciencias sociales ${ }^{5}$. Las claves de interpretación hacen necesarias más pruebas empíricas. La idea que guía este trabajo es que en Rumanía las tendencias de la movilidad deben ser analizada a la luz del significado social que la experiencia de la migración ha desempeñado y desempeña para los ciudadanos rumanos. A pesar del aislamiento sufrido desde el final de la segunda guerra mundial, de hecho, en Rumanía la movilidad de masas tiene unos orígenes arraigados, que se remontan a la época del régimen comunista.

\section{LOS AÑOS DEL SOCIALISMO Y LA MIGRACIÓN INTERNA}

Durante el periodo comunista, Rumania estaba involucrada en una intensa movilidad interna. En los años cincuenta, el país era predominantemente agrícola. En su intento de crear la Societate Socialista Multilateral Dezvoltata $(\mathrm{SSMD})^{6}$, Ceausescu hizo un impresionante plan masivo de industrialización y de reforma agraria. El auge industrial, que sin embargo fue desigual en las diferentes áreas del país y aumentó las desigualdades ya existentes, determinó el movimiento, más o menos forzado, de una parte importante de la población rumana de las zonas rurales a las urbanas. En este contexto de necesidad de mano de obra industrial se insertan también las draconianas medidas de Ceausescu dirigidas a apoyar el crecimiento de la población (por ejemplo, el duro castigo para el aborto o el impuesto sobre el celibato).

En realidad, como señala Cingolani, una gran parte de estos campesinos urbanizados, que generalmente presentaban bajos niveles de educación y sufrían una fuerte discriminación por parte de los empleadores, no se habían desplazado a la ciudad, sino que se trasladaba todos los días de las zonas rurales de residencia a las fábricas de la ciudad, creando el fenómeno peculiar del

\footnotetext{
${ }^{5}$ Véase in particular Sandu 2000, 2004, 2006; Lazaroiu, 2003; Diminescu 2003; Potot 2003; Weber 2004; Vlase 2004.

${ }^{6}$ La Societate Socialista Multilateral Dezvoltata, concepto desarrollado por Ceausescu en 1965 y difundido a través de una propaganda masiva, es un patrón de "sociedad perfecta", basada en el desarrollo industrial y agrícola intensivo, en la economía planificada y centralizada, en la reducción de las diferencias sociales y en la educación técnica de masa orientada a la creación del "hombre nuevo".
} 
navettismo. Este sistema de desplazamientos permitía descargar los costes del desarrollo industrial totalmente en el campo: de hecho, los campesinos "viajeros", no integrados en el tejido urbano y lejos de formar una "nueva clase obrera", no pesaban en el sistema de bienestar y, a menudo, se pagaban ellos mismos los gastos de transporte (Cingolani, 2009: 36-7).

El pueblo se mantuvo, por lo tanto, como marco simbólico de pertenencia para dos millones de campesinos urbanizados y para todos aquellos que practicaban el navettismo; el mantenimiento de las relaciones familiares y comunitarias hicieron posible la construcción de un nuevo espacio social ciudad-pueblo, capaz de resistir también la acción represiva del régimen (Diminescu, 2003).

Al mismo tiempo, Ceausescu puso en marcha un impresionante proceso de colectivización agraria, a través de la expropiación "voluntaria" de las tierras a las familias campesinas y su destino a las nuevas granjas colectivas. A los miembros de las cooperativas agrarias se les otorgaron sólo 15 hectáreas en usufructo para su autoconsumo. La expropiación "voluntaria" es en realidad el resultado de estrategias diferentes, como coerción, propaganda, reorganización de la agricultura de escala, falsas promesas, prácticas abusivas por parte de la nomenklatura; los registros de propiedad fueron retenidos por las autoridades y sólo fueron abierto públicamente en los años noventa (Gambino, Sacchetto, 2007: 26).

Además de la reestructuración del sector agrícolo, la colectivización tuvo consecuencias sociales muy importantes. De hecho, la sustracción de la propiedad de los medios de producción agrícola determinaba la desintegración de las relaciones de solidaridad de la sociedad campesina, el debilitamiento de los lazos con la tierra, la cancelación del know how individual y colectivo, evitando, en última instancia, que se formara una conciencia de clase (ibidem).

La migración rural-urbana ${ }^{7}$ estuvo también acompañada de formas menos incisivas de movilidad rural-rural (de Moldova y Muntenia hacia Constanza, de las regiones pobres del oeste a Timis) y urbana-urbana (con el movimiento de mano de obra de las ciudades a los centros industriales más pequeños y viceversa) y alcanzó los niveles más altos en la década de los ochenta (Figura 1). El primer efecto observado fue el envejecimiento y la feminización de la población agrícola, por lo que el régimen trató de limitar la migración rural, haciendo más difícil la transferencia a los centros urbanos (Cingolani, 2009).

${ }^{7}$ Sobre el fenómeno de la movilidad rural-urbana en las diferentes áreas durante el período 1950-1980, cfr. Sandu, 1984. 
FIGURA 1

Movilidad interna en Rumanía por área (Período 1968-2002) (\%)

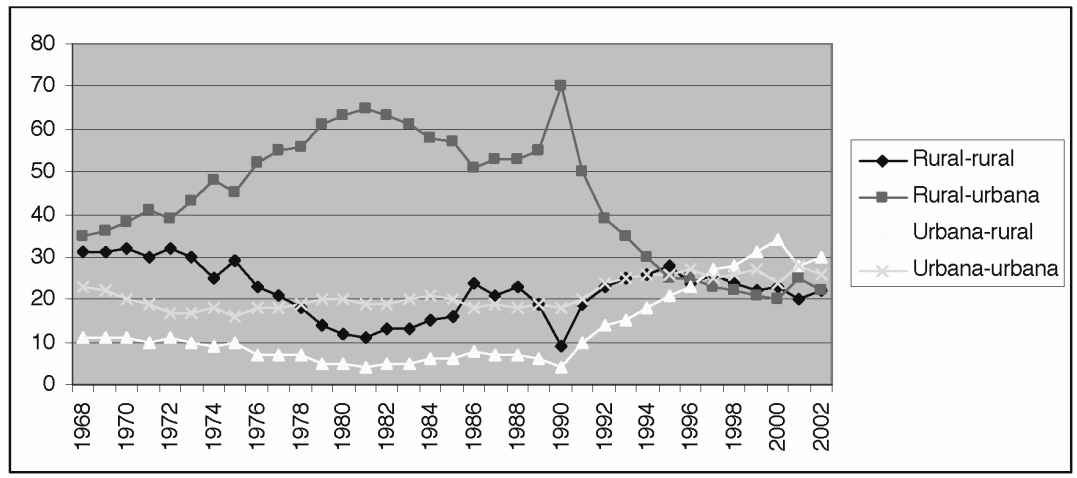

Fuente: Sandu et al., 2004

\section{EL COLAPSO DEL RÉGIMEN}

Con el fin del régimen de Ceausescu en 1989, Rumanía cae en una fase de crisis económica y política sin precedentes. La caída de la producción industrial determina, en unos pocos años, altas tasas de desempleo urbano, un aumento generalizado de los niveles de pobreza y la necesidad de recurrir a estrategias alternativas de supervivencia. Se acentúa la disparidad socioeconómica entre las zonas situadas al oeste del país, caracterizadas por mayores niveles de desarrollo, y el nordeste. La reducción drástica de los recursos para el sistema de bienestar no permite responder adecuadamente a la solicitud de ayuda de la población más vulnerable y a las nuevas necesidades derivadas de los cambios socioeconómicos. Al empobrecimiento material heredado del régimen se añaden nuevas situaciones de malestar social.

La transición a la economía de mercado, un proceso lento, contradictorio y lejos de ser lineal, requiere en primera instancia el desmantelamiento de un sistema centralizado que afecta a todos los aspectos de la vida social y económica del país y la eliminación del aparato institucional de la era comunista. Con la nueva Constitución de 1991 se inicia una política de descentralización administrativa y de reducción de la burocracia en los niveles central y local. Un primer paso es el reconocimiento de 41 distritos como autoridades locales (regiones). Sin embargo, el proceso de reforma es largo y tortuoso.

En los primeros años del post-comunismo, se asiste a un claro cambio de tendencia en la movilidad interna y al gradual desplazamiento de la población desde las zonas urbanas a las zonas rurales (cfr. Sandu 2004 et al.; Rotariu, 
Mezei, 1999)8. El fenómeno del navettismo disminuye considerablemente. Despedidos en masa de las fábricas, los campesinos urbanizados regresan a sus lugares de origen, donde el coste de la vida y los impuestos son más bajos 9 .

Al mismo tiempo, mientras los centros urbanos más pequeños pierden centralidad, las ciudades más grandes (Bucarest, Costanta y Timisoara) se convierten, en cambio, en polos de atracción. Por otra parte, a partir de 1989, los migrantes internos procedentes de las aldeas rurales están oficialmente autorizados a obtener, si quieren, una residencia permanente en la ciudad, a manera de compensación por su migración durante el régimen (Sandu et al., 2004). Para amplios sectores de la población, por lo tanto, trasladarse a las zonas rurales y a las grandes ciudades se convierte en el principal modelo de supervivencia.

La ruralización de parte de la población urbana tiene sus bases en el proceso de descolectivización. Sin embargo, la devolución de las tierras agrícolas a familias campesinas encontró resistencias y dificultades de todo tipo, especialmente debido a la falta de títulos legales de propiedad. Se abrieron causas judiciales que llegan a nuestros días. En 1991, con la promulgación de la Ley 18 sobre la reforma agraria (modificada numerosas veces), se establece la liquidación de las granjas colectivas. Sin embargo, aquellas que resultan más rentables ya habían sido adquiridas por los gerentes del tardosocialismo, y las comisiones encargadas de la privatización, en lugar de devolver las tierras, trataban de compensar a los reclamantes con parcelas de tierra que muy a menudo tenían un valor inferior.

Por lo tanto, en un período de cincuenta años una población tradicionalmente agrícola vio cómo se le quitaba el precioso don de la tierra dos veces: primero con la colectivización, y, a continuación, con la descolectivización (Gambino, Sacchetto, 2007: 26 -27). Frente a la separación forzosa de la tierra, para muchos la alternativa de la emigración al extranjero se convirtió en el único camino viable.

\section{LAS ETAPAS DE LA MIGRACIÓN AL EXTERIOR DESPUÉS DE 1989}

Dana Diminescu (2003) distingue tres etapas de la migración rumana tras la caída del régimen de Ceausescu. La primera fase (1990-1994) está marcada

\footnotetext{
${ }^{8}$ Según Secondo Dumitru Sandu et al. (2004), el año clave en el cual se registra una inversión de tendencia y la migración interna de las ciudades al campo resulta predominante es de 1997. Durante el mismo período hubo un aumento significativo en los niveles de pobreza (del 20\% en 1996 a 31\% en 1997).

${ }^{9}$ Moldavia, por ejemplo, es una de las zonas más afectadas por esta migración de retorno, por primera vez en 1995 con la caída del centro industrial de Brasov, y despues en 1998 con el desmantelamiento de las minas de Hunedoara (Diminescu, 2003).
} 
por un fuerte movimiento a corto plazo hacia los países vecinos. En una situación de caos institucional, económico y social nunca antes experimentado, la población de Rumania, "inocente" en tema de migración internacional, se ve obligada a recurrir a estrategias alternativas de supervivencia, a veces al límite de la legalidad ${ }^{10}$. Entre las diferentes actividades, es particularmente relevante la compraventa de bienes en los países vecinos cruzando (a menudo el mismo día) las fronteras con visados de turista ${ }^{11}$.

Estos migrantes transfronterizos, o "falsos turistas", imitaban el modelo de "los comerciantes con maleta" 12 practicado durante el período comunista por los polacos y, de forma mucho más limitada, por los mismos rumanos, en virtud de acuerdos bilaterales con la vecina Yugoslavia que permitían el "libre" movimiento por un máximo de ocho días al mes sin necesidad de visa o pasaporte ${ }^{13}$. Al mismo tiempo, también comenzó a desarrollarse un tipo de pendularismo transfronterizo por motivos de trabajo (Sandu, 2000; Potot, 2003) ${ }^{14}$.

Junto a estos cambios limitados por el tiempo y apenas visibles, en el mismo período se observa un movimiento masivo de refugiados. De hecho, con la apertura de las fronteras, la primera gran migración a otros países es principalmente de ciudadanos pertenecientes a minorías étnicas y religiosas históricamente presentes en Rumania, que habían estado discriminados por

${ }^{10}$ Por otra parte, un sistema paralelo de economía secundaria para conseguir bienes de primera necesidad, en contraposición al sistema de shortage economy (Kornai, 1992) basado en la racionalización de los bienes por parte del régimen, se había convertido en un componente estructural del sistema económico.

11 Para tener una idea de estos movimientos frenéticos, se estima que en 1992 un millón de rumanos se habían ido a Turquía para motivos de "turismo".

${ }^{12} \mathrm{Al}$ principio, este "comercio con maleta" se localizaba en una franja de mercado gris, caracterizado por bajos costos de ejecución, ya que por un lado estaba libre de impuestos por parte del gobierno y por otro lado estaba fuera del control de la delincuencia organizada que controlaba el mercado negro (Cingolani, 2009: 43).

${ }^{13}$ La historia de la cooperación económica entre Rumanía y Yugoslavia empezó en los años sesenta con el inicio del proyecto binacional "Por?ile de Fier I" y "Por?ile de Fier II" para la construcción de una central hidroeléctrica en el Danubio. El proyecto incluía también un sistema de navegación. Como consecuencia, en 1967 se firmó un acuerdo bilateral que establecía la posibilidad para los ciudadanos de ambos países que vivían en las zonas adyacentes a la frontera de pasar la frontera con sólo un documento (pasul de mic traffic) expedido por las autoridades locales. El propósito declarado de esos viajes era el turismo, pero la mayoría de estos "turistas" se dedicaban a la compra de bienes esenciales disponibles en el mercado local (Sandu et al., 2004).

${ }^{14}$ Durante los años noventa, en virtud de acuerdos bilaterales, comienza también la contratación de trabajadores de temporada en Alemania, Rumania e Israel, aunque de forma muy limitada en comparación con otros países de Europa del Este. En 1993, por ejemplo, fueron reclutados para trabajar en Alemania, 3.853 rumanos (Werner, 1995). 
el régimen, sobre todo alemanes, húngaros y judíos ${ }^{15}$. Entre 1990 y 1993, 325.900 personas solicitaron asilo político en los países occidentales (en particular Alemania), lo que llevó a los gobiernos a adoptar políticas más restrictivas en los años siguientes. Entre estos refugiados, había muchos rumanos de etnia romaní (véase Benattig y Brachet, 1998), que solicitaban protección internacional por la política de asimilación y discriminación del gobierno de Rumania contra ellos.

El flujo migratorio de rumanos de minorías étnicas (húngaros, alemanes, etc.) baja considerablemente desde la mitad de los años noventa, dejando espacio para la migración más allá de la componente étnica mayoritaria de esa población migrante. Cambian también los principales países de destino; Alemania y Hungría son sustituidos por los Estados Unidos y Canadá y, más tarde, por los países del Sur (Sandu et al., 2004).

A una primera fase de movilidad exploratoria y transfronteriza, expresión de un derecho negado por mucho tiempo, sigue una segunda fase (19942000), motivada principalmente por la búsqueda de un puesto de trabajo, bajo la presión de una crisis económica cada vez más fuerte. En un clima de prohibiciones y restricciones a la movilidad fuera de la Comunidad, como resultado de la consolidación de la Fortaleza Europa, estas migraciones laborales a menudo toman la forma de solicitud de asilo o entrada ilegal.

Aprovechando la experiencia pasada de movilidad transfronteriza, los potenciales migrantes rumanos intentan entrar en el espacio Schengen con varios trucos, para luego establecerse ilegalmente en un Estado miembro y buscar una forma de regularización (por ejemplo, a través de una amnistía). En Alemania, por ejemplo, los rumanos de origen alemán que regresaron al país en el período anterior jugaron un papel clave: a través de la práctica de la invitación, se permitió que miles de conciudadanos (vecinos, amigos, familiares,...) se expatriaran. Sin embargo, en virtud de acuerdos firmados por Rumania con la mayoría de los países de la UE muchos irregulares fueron repatriados.

Esta segunda fase de la migración se caracteriza por un fuerte componente regional y por la formación de cadenas migratorias que unen algunos pue-

${ }^{15}$ De hecho, el proceso de expulsión de las minorías étnicas en Rumania ya había sido iniciado por Ceausescu, el cual, según un proyecto de homogeneización nacional, había firmado acuerdos bilaterales con Israel en primer lugar y, a continuación, con Alemania (acuerdo Schmidt-Ceausescu de 1978) para el retorno de los judíos y de los ciudadanos de origen alemán (Aussiedler). Entre 1950 y 1989 más de 242.000 Aussiedler habían salido de Rumania; en los tres años siguientes a la caída del régimen fueron unos 140.000. Los judíos que llegaron a Israel entre 1961 y 1964 fueron casi 64.000; en los años siguientes el número de repatriados se redujo gradualmente y en la actualidad la comunidad judía rumana cuenta con unas 9.000 personas. Los húngaros que regresaron a su país fueron cerca de 25.000 en el período 1987-1989 y casi 32.400 desde 1989 (Diminescu, 2003). 
blos "pilotos" a áreas específicas en los países de destino ${ }^{16}$. Según Dumitru Sandu, los flujos migratorios antes de 2002 proceden principalmente de las zonas menos pobres de Rumania caracterizadas por una alta heterogeneidad cultural, como la parte occidental de Moldavia y el norte de Transilvania. Las tasas son más bajas en las zonas más pobres y más aisladas (Vaslui, Ialomita, Teleorman, Calarasi, Mehedinti, Gorj, Salaj). Los pueblos afectados por la migración temporal en el extranjero generalmente están ubicados cerca de las ciudades pequeñas, tienen una población más joven, con más formación y altos niveles de diversidad cultural (sobre todo religiosa) (Sandu et al., 2004).

En esencia, empieza a definirse un modelo de "migración circular" que, aprovechándose de las fisuras en los aparatos legislativos de los estados nacionales, constituye, como se verá más adelante, la piedra angular del "sistema de migración rumano". Paralelamente, se desarrolla un sistema siempre más eficiente de smuggling y trafficking, dirigido por organizaciones criminales para la entrada ilegal y la explotación de los migrantes en los países de destino ${ }^{17}$

En 2002, con la derogación del visado de entrada en el espacio Schengen, se inaugura una tercera fase migratoria. En el marco de las negociaciones para la adhesión de Rumanía a la Unión Europea, a los ciudadanos rumanos se le permite moverse dentro de la zona con un visado turístico por un período máximo de tres meses. Los vínculos impuestos por el gobierno rumano para frenar la emigración (necesidad de un seguro médico reconocido en el extranjero, billete de ida y vuelta, 100 euros por cada día de estancia y dirección en el país de destino) son fácilmente eludidos mediante el uso de varios trucos (por ejemplo, el soborno a la policía de fronteras). Los flujos migrato-

${ }^{16}$ Los estudios de Diminescu y Lazaroiu (2002) identifican algunas de estos pueblos piloto, en Certeze (en París), Borsa (Milán), Sambata de Sus (en Roma).

17 Según el primer protocolo adicional de la Convención de las Naciones Unidas Contra la Delincuencia Organizada Transnacional (Palermo 2000) por "trata de personas" (trafficking) se entenderá la captación, el transporte, el traslado, la acogida o la recepción de personas, recurriendo a la amenaza o al uso de la fuerza u otras formas de coacción, al rapto, al fraude, al engaño, al abuso de poder o de una situación de vulnerabilidad o a la concesión o recepción de pagos o beneficios para obtener el consentimiento de una persona que tenga autoridad sobre otra con fines de explotación. Esa explotación incluirá, como mínimo, la explotación de la prostitución ajena u otras formas de explotación sexual, los trabajos o servicios forzados, la esclavitud o las prácticas análogas a la esclavitud, la servidumbre o la extracción de órganos (art. 3, lettera a). En el segundo Protocolo Adicional se define el reato de smuggling o "tráfico ilícito de migrantes" como la facilitación de la entrada ilegal de una persona en un Estado Miembro del cual dicha persona no sea nacional o residente permanente con el fin de obtener, directa o indirectamente, un beneficio financiero $\mathrm{u}$ otro beneficio de orden material (letra a). Sobre esta cuestión, Report of the Experts Group on Trafficking in Human Beings, 2004; Mancini, 2008, Baldoni, 2007). 
rios circulares aumentan ${ }^{18}$, incluso con el reclutamiento cada vez mayor de trabajadores de temporada procedentes de Rumanía por otros países europeos (Alemania, España, Portugal, Suiza y Hungría) a través de un sistema de cuotas. De todos modos, España e Italia se fortalecen como destinos preferidos.

Los primeros que toman ventajas del nuevo régimen de circulación son los migrantes rumanos irregulares, que finalmente pueden volver a la patria y, gracias al capital migratorio acumulado, intentan nuevamente dejar el país por medios legales. Además, este sistema facilita la movilidad de otros grupos, especialmente de los familiares (u otros miembros de la comunidad), de los gitanos y de grupos "más visibles, pero poco numerosos", usando las palabras de la misma Diminescu, formado por mujeres, niños y personas con discapacidad, víctimas de trata con fines de explotación en los países de destino.

El crecimiento de las mujeres en estos flujos ha sido constante desde el año 2002, coincidiendo con la creciente demanda de servicio doméstico y ayuda familiar (especialmente en España e Italia). El trabajo de cuidado ha adquirido de hecho una connotación específica étnico-nacional, por lo que las mujeres de Europa Oriental constituyen la mayor parte de trabajadoras domésticas en muchos países occidentales (cfr. Torre et al., 2009) ${ }^{19}$.

La migración femenina es emblemática para la comprensión de los cambios que tienen lugar en la sociedad rumana. Las mujeres deciden dejar el país a pesar de la carga de responsabilidades familiares y sociales (hijos, cónyuge, familia, vecinos...) que les ha asignado un contexto aún profundamente patriarcal; enfrentándose con mentalidades diferentes e imponiendo los tiempos de su presencia y ausencia tanto en el espacio de salida como en el de destino (Gambino, Sacchetto, 2007: 31).

\section{LOS PILARES DEL SISTEMA MIGRATORIO RUMANO}

Después de esta reconstrucción en clave histórica de la migración rumana, lo que demuestra su importancia social y cultural, es necesario focalizar la atención en las dimensiones actuales del fenómeno. Según el último censo de 2002, los rumanos que viven temporalmente en el extranjero son 360.000, de una población de cerca de 21 millones de personas (representa un 17\%) (Sandu et al., 2004). A esto tenemos que añadir los emigrantes "permanentes", que en el período 1990-2009, según datos del Instituto Nacional de Estadística de Rumanía, fueron cerca de 414.600.

\footnotetext{
${ }^{18}$ Según Sandu (2006), desde 2002 la tasa anual de migración temporal ha sido cada vez mayor, pasando de 10 a 28 personas por cada 1.000 habitantes. El mayor incremento se registró en la región de Moldavia, Transilvania y Muntenia.

${ }_{19}$ Sobre la migración de mujeres rumanas Italia, cfr. Castagnone et al., 2007; Vlase, 2006; Licata e Pittau, 2008.
} 
Como se muestra en la tabla 1 , la evolución de las salidas es fluctuante; se observa que los intentos realizados por el gobierno rumano para frenar la emigración después de la supresión de la visa Schengen en 2002, parecen "contener" las salidas. En realidad, como se señaló anteriormente, en este periodo hubo un aumento considerable de la migración irregular.

TABLA 1

Ciudadanos rumanos emigrados al extranjero por sexo (1990-2009) (Flujos)

\begin{tabular}{l|c|c|c}
\hline Año & Total & M \% & F \% \\
\hline $\mathbf{1 9 9 0}$ & 96.929 & 47,8 & 52,2 \\
\hline $\mathbf{1 9 9 1}$ & 44.160 & 48,0 & 52,0 \\
\hline $\mathbf{1 9 9 2}$ & 31.152 & 51,6 & 48,4 \\
\hline $\mathbf{1 9 9 3}$ & 18.446 & 47,4 & 52,6 \\
\hline $\mathbf{1 9 9 4}$ & 17.146 & 46,0 & 54,0 \\
\hline $\mathbf{1 9 9 5}$ & 25.675 & 44,7 & 55,3 \\
\hline $\mathbf{1 9 9 6}$ & 21.526 & 46,8 & 53,2 \\
\hline $\mathbf{1 9 9 7}$ & 19.945 & 47,2 & 52,8 \\
\hline $\mathbf{1 9 9 8}$ & 17.536 & 48,2 & 51,8 \\
\hline $\mathbf{1 9 9 9}$ & 12.594 & 46,5 & 53,5 \\
\hline $\mathbf{2 0 0 0}$ & 14.753 & 46,1 & 53,9 \\
\hline $\mathbf{2 0 0 1}$ & 9.921 & 50,5 & 49,5 \\
\hline $\mathbf{2 0 0 2}$ & 8.154 & 45,4 & 54,6 \\
\hline $\mathbf{2 0 0 3}$ & 10.673 & 41,3 & 58,7 \\
\hline $\mathbf{2 0 0 4}$ & 13.082 & 37,7 & 62,3 \\
\hline $\mathbf{2 0 0 5}$ & 10.938 & 37,6 & 62,4 \\
\hline $\mathbf{2 0 0 6}$ & 14.197 & 37,6 & 62,4 \\
\hline $\mathbf{2 0 0 7}$ & 8.830 & 35,0 & 65,0 \\
\hline $\mathbf{2 0 0 8}$ & 8.739 & 35,1 & 64,9 \\
\hline $\mathbf{2 0 0 9}$ & 10.211 & 36,9 & 63,1 \\
\hline Total & 414.600 & & \\
\hline
\end{tabular}

Fuente: Institutul National de Statistica de Rumania

De hecho, frente a esta migración "registrada", fuentes no oficiales hablan de más de 2 millones de migrantes, lo que representa una quinta parte de la población activa (véase Ricci, 2010). La subestimación del fenómeno por parte de las fuentes oficiales rumanas se debe tanto a la misma consistencia de la 
inmigración ilegal (antes de la entrada en la Unión Europea) como a los movimientos circulares que permanecen en gran parte indocumentados. De acuerdo con un estudio sobre la migración rumana desde 1990 a 2006, un tercio de los hogares tenía (o había tenido en el momento de la encuesta) al menos un miembro en el extranjero por motivos de trabajo (Sandu, 2006). Este resultado es confirmado por otra investigación nacional en 2007, que, a fin de evaluar el interés por la movilidad, también muestra que, en comparación con un 55\% de la población "sedentaria", la proporción de los que expresan la intención de trabajar en el extranjero es dos veces mayor que en el 2006 (Andreescu, Alexandru, 2007).

El "sistema migratorio rumano" parece sentar sus bases en dos pilares. En primer lugar, se basa en la estrecha relación entre movilidad interna (especialmente entre las zonas rurales y urbanas del país), migración permanente (muy relacionada con las minorías étnicas en los primeros años noventa) y migración temporal (Sandu et al., 2004). Según Diminescu (2003), la experiencia de la movilidad interna, que se caracteriza por una circularidad intensa y por mantener fuertes vínculos con la comunidad de origen, ha afectado directamente a las prácticas de la migración internacional de los rumanos y ha ayudado a seleccionar a los candidatos para la salida. Algunos estudios empíricos realizados en Italia, Francia, Alemania e Israel sobre los migrantes que se trasladaron a Rumania en los años 1994-2000 muestran una sobrerrepresentación de los viejos "navettisti", como si este "ir y venir", diluido en el tiempo, se hubiera reproducido en las largas distancias.

La segunda característica distintiva es el predominio de la migración temporal (o circular), "nuevo pilar del sistema migratorio rumano", según Sandu et al. (2004). Esta es una forma compleja y dinámica, en gran parte no cuantificada ni documentada, que se refleja en prácticas transnacionales que involucran la presencia simultánea de los migrantes tanto en el contexto de origen como en el de destino.

En los últimos años se ha desarrollado un amplio debate interdisciplinar sobre el tema del transnacionalismo ${ }^{20}$. El concepto de transnacionalismo, que surgió en el contexto de las ciencias políticas, se introdujo en el campo de la sociología de la migración en los años noventa por Nina Glick Schiller, Basch Linda y Cristina Szanton Blanc como el "proceso por el cual los inmigrantes construyen campos sociales ${ }^{21}$ que unen el país de origen y el de destino"

${ }^{20}$ Cfr. Glick Schiller et al., 1992; Portes, 2001; Portes, 2003; Faist, 2000; Faist, 2008; Guarnizo et al., 2003; Vertovec, 2004; Kivisto, 2001; Levitt et al. 2004; Waldinger, Fitzgerald, 2004; Levitt et al. 2007; Ambrosini, 2007.

${ }^{21}$ El concepto de campo social transnacional se refiere a un network de relaciones centrado en la comunidad, más que sobre el individuo (Wimmer, Glick Schiller, 2003). 
$(1992,1)$. El transnacionalismo no representa un fenómeno nuevo en sí mismo, sino una nueva perspectiva para analizar la dinámica de la migración (o al menos algunos aspectos escondidos o con diversas interpretaciones) que parte "desde lo más bajo", desde experiencias individuales de movilidad que, sin embargo, asumen importancia macrosocial (Portes, 2003).

En abierta oposición al nacionalismo metodológico (Beck, 2005), que estudia los procesos (en este caso) migratorios dentro de los límites de un determinado Estado-Nación, en el paradigma transnacional la migración no se configura como un movimiento lineal desde el país de origen hasta el de destino, sino como un flujo pluri-direccional y continuo de personas, mercancías, capitales e ideas que cruzan las fronteras nacionales conectando diferentes espacios físicos, sociales, económicos y políticos (Cingolani, 2009: 14). La red de intercambios entre dos (o más) países incluye, por lo tanto, tanto el movimiento físico de personas (pendulares) y bienes, como la circulación de bienes inmateriales, tales como dinero, información, mensajes, prácticas sociales, y también patrones culturales, valores, símbolos, estilos de vida y representaciones.

El transmigrante participa simultáneamente en ambos polos del movimiento migratorio (Ambrosini, 2007), operando simultáneamente en diferentes contextos sociales (Boccagni, 2007). Gracias al extraordinario desarrollo de medios de transporte y comunicación (por ejemplo, Internet, tecnología o vuelos low cost), es capaz de llevar a cabo actividades económicas o comerciales, alternar diferentes períodos de empleo, establecer relaciones sociales y afectivas, ejecutar proyectos, gestionar sus propios intereses en una dimensión inter-espacial que trasciende las fronteras políticas, geográficas de los propios Estados. Esto pone en discusión la cuestión de los efectos de la migración internacional y obliga a revisar las categorías tradicionales de "inmigrante" y "emigrante".

La movilidad transnacional no representa simplemente un recurso o una oportunidad de promoción social. Incluso dentro de un espacio transnacional, la experiencia de migración, además de reproducir las desigualdades de partida o fenómenos de pérdida de poder, puede llevar a una condición de exclusión y aislamiento relacional en ambos contextos.

La perspectiva transnacional ofrece una explicación eficaz sobre la reciente migración de Europa del Este post-comunista, que se originó en un clima importante de crisis económica y política. Por otra parte, este modelo responde a características específicas del contexto europeo, tales como la ausencia de condiciones propicias para el establecimiento en el país de destino, la facilidad de los viajes por Europa y, por último pero no menos importante, la reducción de estatus social que el migrante sufre en la sociedad de destino, y 
que le lleva a mantener, durante las distintas fases de trabajo en gran parte no cualificados y precarios, el país de origen como espacio de referencia principal y de inversión (Cingolani, 2009: 18).

En el caso de Rumanía, la migración temporal al exterior ha llegado a ser más consistente con el aumento de los niveles de pobreza, el retorno al campo y la disminución de la migración permanente (Sandu et al., 2004). Desde el año 2002, representa una verdadera "estrategia de vida" para individuos y familias, una estrategia que implica a nivel personal y social la participación en el "saber migratorio" acumulado, el apoyo de las redes étnicas, el desarrollo de una gran capacidad de adaptación y redefinición de las relaciones.

Emblemático es el caso de el share-work, un modelo transnacional de gestión familiar, que le permite también a las mujeres rumanas que tienen hijos de migrar por períodos cortos para trabajar como cuidadoras (a menudo sustituyendo una compañera que ha regresado a Rumania), manteniendo al mismo tiempo su rol dentro del núcleo familiar (Morokvasic, 2003).

\section{LOS RUMANOS EN ITALIA ENTRE CIRCULARIDAD Y ESTABILIZACIÓN}

Según los últimos datos difundidos por ISTAT ${ }^{22}$, los extranjeros que residen legalmente en Italia el 1 enero 2010 son 4.235 .059 (7\% de la población total). En el año 2009, el número de extranjeros aumentó en 343.764 personas $(+8,8 \%)$, un aumento que sigue siendo muy elevado, aunque inferior a los dos años anteriores (494.000 en 2007 y 459000 en 2008, respectivamente $+16,8 \%$ y $13,4 \%)$. Alrededor de la mitad de los residentes extranjeros es originario de países de Europa del Este: en particular, un cuarto de los nuevos miembros de la UE y otro cuarto de los países de Europa del Este fuera de la UE.

La comunidad rumana, con 887.763 personas (de los cuales $53,9 \%$ son mujeres) representa el $21 \%$ de todos los inmigrantes presentes en Italia, siendo el grupo extranjero más numeroso ${ }^{23}$. A nivel territorial, resulta predominante en muchas regiones, incluido Lazio (donde constituye el 36\% del total de residentes extranjeros, es decir, aproximadamente 179.000 personas), Piemonte (34,5\%, más de 130.000 unidades), Lombardía (13,1\%, casi 129.000 personas) y Veneto (20,2\%, casi 97.000 habitantes). Además, teniendo en cuenta la difusión de flujos circulares no documentados ${ }^{24}$, es posible que esta

${ }_{22} \mathrm{Cfr}$. Statistiche in breve, La popolazione straniera residente in Italia al $1^{\circ}$ gennaio 2010, http://www.istat.it (novembre 2010).

${ }^{23}$ Seguidos de Albania (466.684), Marruecos (431.529), China (188.352), Ucrania (174.129).

${ }^{24}$ Para intentar cuantificar las migraciones circulares, el Tercer Informe EMN Italia (EMN, 2010) ha editado un análisis de los archivos estadísticos. Además de las estadísti- 
proporción supere el millón. Según las estimaciones del Dossier Caritas/Migrantes, los rumanos que realmente permanecen en Italia serían 1.110.0001.165.000, tomando en cuenta un $12 \%$ "indocumentados" que todavía están esperando para ser inscritos en las listas de los registros municipales (Ricci, 2010).

En cuanto a los flujos anuales "oficial" (véase la tabla 2) se observa que, si por un lado la tendencia de ingresos procedentes de Rumanía sigue creciendo, por otro lado, el aumento registrado en 2009 (igual a 11, 5\%) resulta significativamente inferior al del año anterior (igual a 27,4\%). Esto permite deducir que el "impulso migratorio" producido por la entrada del país en la Unión Europea está disminuyendo significativamente. También es importante señalar que el aumento de $82,7 \%$ de los rumanos tras la ampliación de 2007 se refiere, con toda probabilidad, principalmente a personas ya presentes en el territorio, invisibles en las estadísticas oficiales al encontrarse como irregulares.

Es posible en este punto dar una respuesta parcial a una de las preguntas iniciales. En Italia, uno de los destinos favoritos de la movilidad de Rumania, la temida "invasión" a raíz de la entrada de Rumanía en la Unión Europea no parece haber ocurrido (y esto a pesar del hecho de que las restricciones previstas en el régimen transitorio fueron más bien suaves). La explicación de la presencia sustancial de los rumanos (presencia que crece a lo largo de la década) no se agota en el factor de la "libre" movilidad y debe buscarse en otra parte.

En cuanto a los otros países receptores, aunque en Hungría y Alemania el porcentaje de rumanos ha tenido durante la década también oscilaciones negativas (aunque nunca haya superado más que las 100.000 personas), España, con sus 799.225 rumanos registrados en 2009, muestra un patrón similar al de Italia.

Según la encuesta periodica de ISTAT sobre la fuerza de trabajo de 2009 (véase el informe EMN, 2010), en Italia los trabajadores inmigrantes son casi dos millones (1.898.000) y representan aproximadamente el 10\% de los empleados (en cambio, los que no tienen un permiso de residencia serían, según las estimaciones, alrededor de medio millón). La contratación programada de trabajadores desde el extranjero fue de 168.000 en 2008, 89.000 en

cas sobre los flujos estacionales, otras fuentes posibles son los archivos del Ministerio de Relaciones Exteriores (los que muestran que los inmigrantes que llegaron en 2009 para un período de tres meses fueron 31.394), los datos ISTAT sobre las cancelaciones de los extranjeros en los registros municipal (más de 32.000 en 2008), los datos del Ministerio del Interior sobre los permisos de residencia caducados (alrededor de 100.000). 


\section{TABLA 2}

Ciudadanos rumanos residentes en el extranjero por los primeros cuatro países de destino (v.a.) y variación porcentual respecto al año anterior (2000-2009) (Stocks)

\begin{tabular}{l|r|r|r|r|r|r|r|r|r|r|r}
\hline & 2000 & 2001 & 2002 & 2003 & 2004 & 2005 & 2006 & 2007 & 2008 & 2009 & 2010 \\
\hline España & 5.682 & 26.779 & 53.087 & 112.861 & 189.979 & 287.087 & 388.422 & 539.507 & 734.764 & 799.225 & - \\
var \% & - & 371,3 & 98,2 & 112,6 & 68,3 & 51,1 & 35,3 & 38,9 & 36,2 & 8,8 & - \\
\hline Alemania & 87.504 & 90.094 & 88.102 & 88.679 & 89.104 & 73.365 & 73.043 & 78.452 & 90.614 & 100.429 & - \\
var \% & - & 3,0 & $-2,2$ & 0,7 & 0,5 & $-17,7$ & $-0,4$ & 7,4 & 15,5 & 10,8 & - \\
\hline Hungría & 57.343 & 43.165 & 44.977 & 47.281 & 55.676 & 67.608 & 66.250 & 66.951 & 65.903 & 66.435 & - \\
var \% & - & $-24,7$ & 4,2 & 5,1 & 17,8 & 21,4 & $-2,0$ & 1,1 & $-1,6$ & 0,8 & - \\
\hline Italia & 41.587 & 62.262 & 74.885 & 95.039 & 177.812 & 248.849 & 297.570 & 342.200 & 625.278 & 796.477 & 887.763 \\
var\% & - & 49,7 & 20,3 & 26,9 & 87,1 & 40,0 & 19,6 & 15,0 & 82,7 & 27,4 & 11,5 \\
\hline
\end{tabular}

Fuente: Elaboración sobre datos Eurostat; Istat

2009 (debido a la crisis) para subir en 2010 a 181.000. Los sectores más deficientes de mano de obra son los relativos a la construcción, hoteles y restaurantes, servicios de salud, servicios a las empresas y agricultura. Los inmigrantes presentan una tasa de actividad superior a los italianos $(72,7 \%$ vs $64,4 \%)$ pero, debido a la crisis, tienen tambíen una tasa de desempleo más alta (11,2\%, 3,5\% más que los indígenas). La mayor ocupación está ligada por un lado a la edad media más joven de la población migrante, por otro lado a la propensión de los extranjeros a aceptar empleos de baja cualificación (generando el fenómeno del brain waste).

En el contexto general del mercado de trabajo italiano, los rumanos jugan un papel muy importante. Según los datos disponibles, en 2008 la contratación de rumanos fue de 174.500 (40\% del total). Estas contrataciones se hicieron casi un tercio en la industria (en particular construcción), la mitad en los servicios (atención a la familia, hoteles y restaurantes, informatica y servicios a las empresas y un quinto en la agricultura (Ricci, 2010). En general, los trabajadores de origen rumano presentan una distribución por sector productivo en línea con la del total de los trabajadores extranjeros (tabla 3). La más importante diferencia se refiere al sector de la construcción, donde hay una concentración mayor del $8,5 \%$. Además, esta comunidad es muy activa en la creación de empresas (aproximadamente 9.000 por año), por lo que en mayo de 2009 habían 28.000 empresas con el empresario de nacionalidad rumana, especialmente en el sector de la construcción (en el segundo lugar después de los marroquíes). 
TABLA 3

Ocupación de trabajadores rumanos por sectores (2009) (\%)

\begin{tabular}{l|r|r}
\hline \multicolumn{1}{c|}{ Sector } & $\begin{array}{c}\text { \% trabajadores } \\
\text { rumanos }\end{array}$ & $\begin{array}{c}\text { Total trabajadores } \\
\text { extranjeros }\end{array}$ \\
\hline Agricultura & 4,6 & 4,0 \\
\hline Industria & 45,4 & 40,4 \\
En sentido estricto & 18,8 & 21,1 \\
Construcción & 26,6 & 18,1 \\
\hline Servicios & 50,0 & 55,6 \\
Comercio & 5,5 & 8,9 \\
Hoteles y restaurantes & 7,5 & 8,4 \\
Servicios para las familias & 23,0 & 21,5 \\
\hline Total & 100,0 & 100,0 \\
\hline
\end{tabular}

Fuente: Informe EMN (2010) sobre datos ISTAT, Rilevazione sulle forze di lavoro

El dinamismo de los trabajadores procedentes de Rumanía en el sistema de producción italiano indica un buen nivel de integración económica. Sin embargo, la adquisición del status de ciudadanos de la UE, que tienen pleno derecho a la libre circulación, debería haber dado lugar a la completa supresión de las irregularidades. En cambio, todavía quedan problemas de trabajo negro o no declarado (sobre todo en el ámbito de la atención familiar, la agricultura y la construcción) ${ }^{25}$, dificultad de acceso a la vivienda y a los servicios básicos, situaciones de pobreza y exclusión social (por ejemplo, la compleja cuestión los rumanos de origen rom), hasta casos de grave explotación laboral y trata de blancas.

No se puede, por otra parte, no mencionar el clima de hostilidad e intolerancia que en el período 2007-2008 se ha creado en Italia hacia la comunidad rumana. A raíz de unas graves noticias de los cuales han sido protagonizadas por ciudadanos rumanos ${ }^{26}$, se dedicó un amplio espacio en los medios de comunicación una campaña agresiva centrada en la supuesta relación entre delincuencia y presencia de rumanos, campaña que también superponía en manera totalmente instrumental "rumanos" y "rom" 27 . El debate sobre

\footnotetext{
${ }^{25}$ Por otra parte, el trabajo irregular, endémico en algunos sectores, presenta todas las ventajas de la deslocalización pero sin suponer un coste adicional a la empresa (Cingolani, 2009).

${ }^{26}$ Se hace referencia, en particular al brutal asesinato de una mujer italiana en un barrio de Roma en octubre de 2007.

${ }^{27}$ Sobre este aspecto, cfr. Harja, Melis, 2010.
} 
la "cuestión rumana" se culminó después con una serie de medidas legislativas que introducían la posibilidad de expulsión de ciudadanos de la UE por razones de seguridad pública ${ }^{28}$.

Frente a esto, otros indicadores muestran una positiva integración de la comunidad rumana en la sociedad italiana. El dato más interesante se refiere a "los nuevos italianos", los jóvenes de segunda generación. En un país cada vez más viejo y destinado al declive demográfico, en 2009 los menores de origen extranjero resultan ser, según el ISTAT, 932.675 (el 22\% de la población extranjera), de los cuales 572.720 nacieron en Italia (sólo 77.148 en ese año). Rumania es uno de los principales países de origen. Y, sin embargo, de acuerdo con datos del Ministerio de la Educación, en el año escolar 2009/2010, 673.592 estudiantes extranjeros asistieron a la escuela italiana. Los alumnos rumanos son más frecuentes en todos los niveles escolares, aunque la tasa anual de crecimiento parece haberse estabilizado. Al mismo tiempo, aumentan la matriculación de los rumanos en la Universidad.

Otro indicador importante de integración se refiere al aumento de los matrimonios mixtos. En 2008, de más de 246.600 matrimonios celebrados en Italia, el $15 \%$ era representado por parejas mixtas, formadas principalmente por hombres italianos y novias de países con fuerte presión migratoria. Una vez más, la principal nacionalidad de las esposas extranjeras es Rumana ( 2.506 casos). Sin embargo, este dato debe ser interpretado con cuidado/precaución: en efecto, en términos absolutos, el tipo inverso "mujer italiana esposo rumano" es mucho menos frecuente. Vale la pena señalar, por último, el aumento de las compras de imuebles; en 2007 sólo en Roma fueron 10.000 las compras de viviendas por los rumanos (Caritas/Migrantes, 2008).

Entonces, ¿cuál es la tendencia predominante de la migración rumana en Italia, entre circularidad y estabilización? El caso italiano parece caracterizarse por la coexistencia de ambos fenómenos. El empuje a la movilidad tempo-

\footnotetext{
${ }^{28}$ En concreto, para abordar el "problema rom", fue promulgado el D.Ley 249 del 29 de diciembre de 2007, para facilitar la expulsión de ciudadanos de terceros países y comunitarios por razones de seguridad. Posteriormente, el decreto del Presidente del Consejo de Ministros de 21 de mayo de 2008 (modificado por tres ordenes del 30 de mayo, 2008 No 3676, 3677 y 3678) declaraba un "estado de emergencia en relación con los asentamientos de las comunidades gitanas en Campania, Lombardía y Lazio" hasta el 31 mayo de 2009 y atribuiba a los funcionarios de las agencias estatales y locales facultades extraordinarias, concebibles sólo en el caso de grandes desastres naturales. A raíz de estas medidas, dirigidas directamente a los miembros de la etnia romaní y sinti, independientemente de su nacionalidad, los que viven en campamentos en Nápoles, Roma y Milán han sido censados voluntariamente tomandoles las huellas digitales. Por estas medidas, que violan el principio de no discriminación, Italia ha sido condenada por varios organismos internacionales y por la misma Unión Europea (sobre esta cuestión, cf. Cherchi, Loy, 2009).
} 
ral madura en un contexto de origen en transición dominado por inseguridad y inestabilidad, que genera fuerte desorientación. Como observa Cingolani, "Rumania contemporánea puede ser descrita como un mosaico de subjetividades empeñados en una cotidiana construcción de identidad, a menudo solitaria, porque no existen panoramas de referencia, solidaridades cohesivas, movimientos sociales capaces de incluir" (2009: 31). La precariedad del trabajo en Italia (potenciado por la crisis económica) aumenta sin duda la temporalidad de la migración laboral, a veces dando a la luz ideas originales, como la del share-work en el sector doméstico.

$\mathrm{Al}$ mismo tiempo, los factores antes mencionados indican una propención creciente a la estabilización en el mediano/largo plazo para una parte importante de esta comunidad. Sin embargo se ha observado que los protagonistas de estos procesos son en su mayoría familias rumanas presentes en Italia antes de la adhesión a la Unión Europea mientras que las estrategias de movilidad en el corto plazo son más frecuentes en los "nuevos" inmigrantes (Torre et al. 2009).

Dicho esto, cabe preguntarse, ¿se puede poner en cuestión/discusión el marco de referencia? Es obvio que entre las dos metacategorias "migración permanente" y "migración temporal" hay muchas formas intermedias de movilidad (Gambino, Sacchetto, 2007: 31), estrategias complejas que se desarrollan in itinere, influenciadas por vinculos contextuales, recursos disponibles, sino también deseo de (auto) realización y socialidad. Y antes de nada hay una movilidad vinculada con la adquisición de la ciudadanía europea, que significa construcción y reconocimiento de una identidad europea que interrumpa, al menos en términos formales, la consideración de los demás "extranjeros". El pleno reconocimiento de los rumanos como "comunitarios" (como los alemánes, francéses, españoles...) no es sólo una cuestión juridica de estatus incompleto; implica un cambio de perspectiva que obliga a repensar también las relaciones de poder entre los diferentes estados miembros. Sólo entonces será posible sustituir definitivamente el término migración con movilidad.

\section{NOTAS FINALES. RUMANÍA "PUERTA DE EUROPA": EFECTOS DE LA MOVILIDAD Y TRANSFORMACIONES INTERNAS}

Una última observación se refiere a las consecuencias de la movilidad en el contexto de origen. Según lo descrito antes, desde 1989 la migración interna, transfronteriza e internacional (tanto permanente como temporal) ha transformado profundamente el país. No cabe duda de que la experiencia migratoria aumenta el capital económico, social y cultural de una comunidad, 
generando procesos de desarrollo e innovación. Y en la peculiaridad de movimientos circulares, que implican, sin embargo, una "presencia virtual" de los migrantes, se amplifican las posibilidades de intercambio de información, know how, habilidades, capacidad de crear y mantener redes.

Entre los beneficios materiales de la migración, las remesas juegan un papel fundamental. En 2007, según estimaciones del Banco Nacional rumano, la transferencia de dinero por parte de los rumanos en el extranjero, especialmente de España e Italia, fue de 7.160.000.000 (con un crecimientodel $29,13 \%$ respecto a 2006), igual a el 5\% del PIB (véase Torre et al., 2009). Además de aumentar los niveles de ingresos y bienestar de los hogares (y, en consecuencia, el consumo y la inversión), las remesas son el elemento clave que explica la elección de la separación y que justifica los costos emocionales y sociales de la salida (especialmente para las mujeres).

Teniendo en cuenta estos efectos positivos, los riesgos de una emigración "no planificados" han sido señalados por varias partes. En primer lugar, a nivel demográfico, la salida de más de 2 millones de personas a una edad joven puede causar en el mediano/largo plazo un desequilibrio de la población hacia la edad avanzada, especialmente en presencia de bajas tasas de natalidad. El proceso de envejecimiento es particularmente evidente en las zonas rurales del país (Ghetau, 2007). La contracción de la población en edad laboral ha creado también graves desequilibrios en el mercado del trabajo y una endémica falta de mano de obra interna. El gobierno rumano estima un necesidad de alrededor de medio millón de trabajadores (especialmente en la construcción, industria ligera, industria alimentaria, comercio, turismo, y más recientemente en el sector de la salud, donde el fenómeno del bran drain ha sido muy marcado) y está tratando de poner en marcha medidas para fomentar el retorno de migrantes rumanos (Torre et al., 2009).

Al mismo tiempo, la escasez de mano de obra en la última década ha atraído un flujo creciente (aunque todavía limitado) de trabajadores procedientes de contextos más pobres, en particular de Turquía, República de Moldova, Ucrania y China ${ }^{29}$. Rumania, nueva puerta oriental de entrada a la Unión Europea, representa para estos migrantes el acceso a mejores niveles de vida, aunque los niveles salariales siguen siendo muy bajos y hay amplia difusión del trabajo ilegal. La transformación gradual en país de destino se refleja en el intento del gobierno de gestionar los flujos, en primer lugar ajustando el marco normativo sobre las entradas de ciudadanos extranjeros adecuándolo

${ }^{29}$ En este marco, están apareciendo numerosos casos de trata de seres humanos con fines de explotación sexual y laboral en Rumania. Sobre este aspecto, cf. datos de la Agencia Nacional contra la Trata de Personas Rumanía (ANITP) (http://anitp.mai.gov.ro/). 
a la legislación de la $\mathrm{UE}^{30}$. La tendencia de las entradas, de acuerdo con el Instituto de Estadística de Rumania fue aún fluctuante, con picos en el 1998 (11.907 entradas) y luego de 2008 (10.030); en 2009, con la crisis económica, los inmigrantes fueron 8.606 .

Otro aspecto que vale la pena al fin mencionar, se refiere al impacto social de las salidas sobre los hijos menores que se han quedado en casa. En Rumania, de acuerdo con la Autoridad para la Protección de los Derechos del Niño (ANPDC) al final de junio de 2007 habían 82.464 niños left behind. Un estudio más reciente realizado por UNICEF Rumania estima unos 350.000 niños rumanos con uno o ambos padres en el extranjero, sobre todo residentes en la región de Moldavia. El padre esta ausente con más frecuencia (aproximadamente 157.000 casos) mientras que los niños que han visto emigrar ambos padres son alrededor de 126.000 (véase Torre et al., 2009). Al igual que en otras familias disfuncionales, los riesgos derivados de la falta de figuras parentales han sido identificados principalmente en los trastornos psicológicos, bajo rendimiento escolar y baja asistencia a la escuela.

¿Qué futuro, pues, para la movilidad de Rumania? En estos tiempos de incertidumbre, es difícil de responder independientemente de cómo evolucionan las economías nacionales y mundial. La tendencia más probable es hacia una estabilización de los flujos, al menos en los principales países de recepción. Una reflexión teórica sobre el peculiar "caso rumano" se puso en marcha; el análisis del pasado ayuda a evidenciar los costos y los riesgos de la movilidad y ofrece preciosas indicaciones de política migratoria. La esperanza es que la adquirida ciudadanía europea se convierta pronto en un derecho pleno, sin restricciones legales y culturales.

\section{BIBLIOGRAFÍA}

AMBROSINI, M. (2007). "Prospettive transnazionali. Un nuovo modo di pensare le migrazioni?". Mondi Migranti, 2, 43-90.

ANDREESCU, V., ALEXANDRU, V. (2007). "Transnational Labor Mobility of Romanians: Empirical Findings on Recent Migratory Trends". Journal of Identity and Migration Studies, vol. 1, 2.

BALDONI, E. (2007). Raccanti di trafficking. Una ricerca sulla tratta delle donne straniere a scopo di sfruttamento sessuale. Franco Angeli, Milano.

BECK, U. (2005). Lo sguardo cosmopolita, Carocci, Roma.

BENATTIG, R., BRACHET, O. (1998). "Dynamiques (Les) migratoires roumaines: le cas des demandeurs d'asile en France". Migrations études, 81, 12.

30 Para una revisión de las medidas legislativas cfr. Podariu, Loncier, (2008) y Reprezentare sindicala pentru muncitorii imigranti din Romania (2010). 
BOCCAGNI, P. (2007). "Come si "misura" il transnazionalismo degli immigrati? Dalle teorie alla traduzione empirica: una rassegna metodologica". Mondi Migranti, 2, 109-128.

CARITAS ITALIANA - CARITAS ROMANIA (2010). I romeni in Italia tra rifiuto e accoglienza. Edizioni Idos, Roma.

CARITAS/MIGRANTES (2008). Immigrazione, Dossier Statistico 2007. Idos, Roma.

CARITAS ITALIANA (2008). Romania. Immigrazione e lavoro in Italia. Idos, Roma.

CASTAGNONE et al. (2007). Madri migranti. La migrazione di cura dalla Romania e dall'Ucraina in Italia: percorsi ed impatto sui paesi di origine. CeSPI Working Paper, 34/2007, http://www.cespi.it/WP/WP34\%20Madri\%20migranti.pdf

CHERCHI, R., LOY, G. (a cura di) (2009). Rom e Sinti in Italia. Tra stereotipi e diritti negati. Ediesse, Roma.

CINGOLANI, P. (2009). Romeni d'Italia: migrazioni, vita quotidiana e legami transnazionali. Il Mulino, Bologna.

D'ANGELO, A. (2008). "Le migrazioni romene nell'Unione Europea. Flussi e caratteristiche". In Caritas Italiana, Romania. Immigrazione e lavoro in Italia (pp. 24-33). Idos, Roma.

DIMINESCU, D., LAZAROIU, S. (2002). La circulation migratoire de Roumains après 1989. Comportements migratoires, pratiques institutionnelles et politiques de gestion de la mobilité des Roumains à l'étranger. IOM, Bucarest.

DIMINESCU, D. (Eds.) (2003). Visibles mais peu nombreux. Les circulations migratoires roumaines. Edition de le Maison des Sciences de l'Homme, Paris.

EMN - EUROPEAN MIGRATION NETWORK/MINISTERO DELLINTERNO. (2010). Mercato occupazionale e immigrazione. Edizioni Idos, Roma.

FAIST, T. (2000). The Volume and Dynamics of International Migration and Transnational Social Spaces. Clarendon, Oxford.

FAIST, T. (2008). "Towards Transnational Studies: World Theories, Transnationalism and Changing Institutions". Paper presented at IMISCOE joint conference Diaspora and Transnationalism. Conceptual, Theoretical and Methodological Challenges, 10-11 April, European University Institute, Florence.

GAMBINO, F., SACCHETTO D. (a cura di) (2007). Un arcipelago produttivo: imprenditori e migranti tra Italia e Romania. Carocci, Roma.

GLICK SCHILLER, N., BASCH L.G., BLANC SZANTON, C. (Eds) (1992). Towards a transnational Perspective on Migration. New York Academy of Science, New York.

GHETAU, V. (2007). "Copii care ne lipsesc si viitorul populatiei Romaniei". Sociologie Romaneasca, 5, 2, 7-84.

GUARNIZO, L.E., PORTES, A., HALLER, W. (2003). "Assimilation and transnationalism: Determinants of Transnational Political Action among Contemporary Migrants". American Journal of Sociology, 108, 6, 1211-1248.

HARJA, A., MELIS, G. (2010). Romeni. La minoranza decisiva per l'Italia di domani. Rubettino, Catanzaro.

KIVISTO, P. (2001). "Theorizing Transnational Migration: A Critical Review of Current Efforts". Ethnic and racial studies, 24, 4, 549-577. 
KORNAI, J. (1992). The Socialist System. The Political Economy of Communism. Clarendon Press, Oxford.

LAZAROIU, S. (2003). "More 'out' then 'in' at the crossroads between Europe and the Balkans". Migration Trends in Selected Applicant Countries, vol. IV, Romania, OIM.

LEVITT, P., GLICK SCHILLER, N. (2004). "Conceptualizing Simultaneity: A transnational Social Field Perspective on Society". International Migration Review, 37, 3, 1002-1039.

LEVITT, P., DEWIND J., VERTOVEC, S. (Eds.) (2003) "Transnational Migration: International Perspectives". International Migration Review, Special Issue, vol. 37, n. 3.

LEVITT, P., JAWORSKY, B.N. (2007). "Transnational Migration Studies: Past Development and Future Trends". Annual Review of Sociology, 33, 129-156.

LICATA, D., PITTAU, F. (2008). "Le donne romene tra famiglia e lavoro". In Caritas Italiana, Romania. Immigrazione e lavoro in Italia (pp. 116-125). Idos, Roma.

MANCINI, D. (a cura di) (2008). Traffico di migranti e tratta di persone. Tutela dei diritti umani e azioni di contrasto. Franco Angeli, Milano.

MARIN, M., SERBAN, M. (2008). Trends in the Field of Social Policies and Welfare Reforms in Romania, CeSPI, Background Report, 1/2008, http://www.cespi.it/WPMIG/ BREPORT\%20Romania.pdf.

MOROKVASIC, M. (2003). "Transnational Mobility and Gender: A View from PostWall Europe". In Umut E., Morokvasic M., Shinozaki K. (a cura di), Crossing Borders and Shifting Boundaries (pp. 101- 133). Vol. I, Gender on the Move, Opladen, Leske Budrich.

MOROKVASIC, M. (2004). "Settled in Mobility: Engendering Post Wall Migration in Europe". Feminist Review, vol. 77, n. 1, 7-25.

NANNI, M.P. (2008). "I romeni nel mondo del lavoro italiano all'indomani dell'ingresso nell'UE: un primo bilancio quantitativo". In Caritas Italiana, Romania. Immigrazione e lavoro in Italia (pp. 94-103). Idos, Roma.

PODARIU, E., LONCIER, A. (2008). "Immigrazione e asilo nella Romania dell'allargamento: flussi in transito e bisogno di nuova forza lavoro". In Caritas Italiana, Romania. Immigrazione e lavoro in Italia (pp. 46-50). Idos, Roma.

PORTES, A. (2001). "New Research and Theory on Immigrant Transnationalism, special issue". Global Networks, 1, 3.

PORTES, A. (2003). "Conclusion: Theoretical Convergencies and Empirical Evidence in the Study of Immigrant Transnationalism". International Migration Review, 37 , 3, 874-892.

POTOT, S. (2003). Circulation et réseaux des migrants roumains: une contribution a l'étude des nouvelles mobilités en Europe. UFR Letteres, Arts et Sciences Humaines, Université de Nice-Sophia Antipolis.

REPREZENTARE SINDICALA PENTRU MUNCITORII IMIGRANTI DIN ROMANIA (2010). Studiu asupra imigratiei in Romania. Descriere, analiza, recomandari. Bucureflti. 
RICCI, A. (2010). "Romania: immigrazione e lavoro in Italia prima e dopo l'allargamento". In Caritas Italiana - Caritas Romania, I romeni in Italia tra rifiuto e accoglienza (pp. 14-27). Edizioni Idos, Roma.

ROMAN, D. (2003). Fragmented Identities. Popular Culture, Sex and Everyday Life in Postcommunist Romania. Lexington Books, Lanham.

ROTARIU, T., MEZEI, E. (1999). "Aspects of the Recent Internal Migration in Romania”. Romanian Sociology, n. 1.

ROTH, M., DOBRE, S. (2005). Labour Supply in Care Services. National Report Romania. European Foundation for the Improvement of Living and Working Conditions.

Report of the Experts Group on Trafficking in Human Beings (Brussels, 22 December 2004), documento in inglese scaricabile dal seguente URL: http://ec.europa.eu/ justice_home/doc_centre/crime/trafficking/doc/report_expert_group_1204_en.pdf

SANDU, D. (1984). Fluxurile de migratie in Romania. Editura Academiei, Bucureflti.

SANDU, D. (2000). "Migratia circulatorie ca strategie de viata". Sociologie Romaneasca, 2.

SANDU, D. (2003). "Migratia ca fenomen de retea si de dezvoltare". Sociabilitatea in spatiul dezvoltarii. Polirom, Iasi.

SANDU, D. et al. (2004). A Country Report on Romanian Migration Abroad: Stocks and Flows After 1989. Multicultural Centre Prague, www.migrationonline.cz (nov 2010).

SANDU, D. (2006). Locuirea temporara in strainatate. Migratia economica a romanilor: 1990-2006. Soros, Bucarest.

TORRE, A.R., BOCCAGNI, P., BANFI, L., PIPERNO, F. (2009). Migrazione come questione sociale. Mutamento sociale, politiche e rappresentazioni in Ecuador, Romania e Ucraina. CeSPI Working Papers 57/2009.

UNDP. (2007). National Human Development Report - Romania. Fostering Human Development by Strengthening the Inclusiveness of the Labor Market in Romania. UNDP, Bucarest.

VERTOVEC, S. (2004). "Migrant Transnationalism and Modes of Transformation". International Migration Review, 38, 2, 970-1001.

VLASE, I. (2004). "Femmes et hommes in migration. D'un village Rumain à Rome". Migrations Societé, vol. 16, 93-94.

VLASE, I. (2006). "Donne rumene migranti e lavoro domestico in Italia". Studi Emigrazione, vol. 43, 161, 6-22.

WALDINGER, R., FITZGERALD, D. (2004). "Transnationalism in Question". American Journal of Sociology, 109, 5, 1177-1195.

WEBER, S. (2004). "De la chiane migratoire à la migration individuelle des Roumains à Rome". Hommes et migrations. n. 1250, juillet - aout, 38-49.

WERNER, H. (1995). "Temporary Migration for Employment and Training Purposes and Relevant International Agreements". Select Committee of Experts on ShortTerm Migration, Council of Europe, Strasbourg.

WIMMER, A., GLICK SCHILLER, N. (2003). "Methodological Nationalism, the Social Sciences, and

the Study of Migration: An Essay in Historical Epistemology". International Migration Review, vol. 37, n. 3, 576-610. 